\title{
Using Social Sensing to Understand the Links Between Sleep, Mood, and Sociability
}

\author{
Sai T Moturu*, Inas Khayal ${ }^{\dagger *}$, Nadav Aharony*, Wei Pan* and Alex (Sandy) Pentland* \\ ${ }^{*}$ MIT Media Lab \\ Cambridge, MA 02124 \\ Email: (smoturu,ikhayal)@mit.edu, (nadav,panwei,pentland)@media.mit.edu \\ ${ }^{\dagger}$ Masdar Institute of Science and Technology \\ Abu Dhabi, UAE
}

\begin{abstract}
In recent years, reality mining experiments have provided several novel insights into human social behavior that would not have been possible without the novel use of smartphone sensing. In this work, we leverage the latest reality mining experiment to study social behavior from a public health perspective. In particular, we focus on sleep and mood as they have a considerable public health impact with serious societal and significant financial effects. We endeavor to explore and uncover the associations between sleep, mood and sociability by studying a population of healthy young adults going about their everyday life. We find significant associations between sleep and mood, reiterating observations in the literature. More importantly, we find that individuals with lower overall sociability tend to report poor mood more often, a statistically significant observation. In addition, we also uncover associations between daily sociability and sleep, a previously unreported observation. These results demonstrate the potential of reality mining studies for studying the sociological aspects of significant public health problems. Further, we hope that our work will provide the impetus for larger studies validating some of these observations and ultimately result in behavioral interventions that can improve public health through better social interaction.
\end{abstract}

\section{INTRODUCTION}

The effects of social interactions and social support on health and well-being, both physical and mental, as well as their role in health promotion have been well-documented over the years [1], [2], [3], [4], [5], [6]. The impact of the quantity and frequency of social interactions have been studied in multiple contexts ranging from involvement in crime [7], [8] to stock market participation [9] to choosing health insurance [10]. There is a recent spurt of interest in this space after studies suggesting that social ties (self-reported friends) can have a significant effect on the spread of healthrelated behaviors such as obesity [11] and sleep loss [12].

While past studies have been insightful and have provided some validation of the popular notions, they are driven by selfreported and observational data that are unable to capture the rich and dynamic nature of social interactions in the real world. However, this reliance on such static data was unavoidable due to practical limitations with respect to gathering dynamic social interactions. However, current advances in sensing technology allow us to overcome some of these limitations.

Until the start of this century, most of the data collected on human interactions was through self-reported surveys and experience sampling. More recently, long term monitoring has been implemented using a variety of technologies including video [13], smartphones [14], [15], [16] and other wearable sensing devices [17], [18], [19]. These studies clearly depict the importance of dynamic data collected from the real world and have provided several novel insights into human behavior and sociology.

In this work, we gather such dynamic and continuous data from subjects in a co-located community over a period of few months, alleviating some of the shortcomings of the social and psychological experiments discussed above. Such dynamic data can be obtained through a combination of mobile wireless sensing and regular surveys. Current smartphones are increasingly used as social sensors through several reality mining studies [15], [20], [21]. We believe that such rich information of quantified face-to-face social interactions will provide novel insights. Our recent work [20], [22] validates this approach with observations that such interactions seem to show stronger correlations with behavioral effects including weight gain as opposed to self-reported social contact information. In addition to wireless sensing, traditional survey methods can provide useful information that might not be easy to gather automatically. Such surveys can enable us to track mood and behavioral aspects over many months. Using this integrated approach to social computing, we explore the relationships among social interactions, mood and sleep.

\section{BACKGROUND AND MOTIVATION}

Social interactions and their effects on well-being and life satisfaction have been studied consistently over the last three decades. While their impact on the everyday life of the public is apparent, there is great scope for conveying this message to the public and using this knowledge to develop interventions that can help individuals improve their personal health and wellness. In this work, we focus on two issues with a considerable public health impact - sleep and mood. We detail a pilot study designed to explore the relationship of sleep and mood with quantified real-world social interactions and with each other.

It is estimated that 50-70 million Americans suffer from a chronic disorder of sleep and wakefulness [23]. Sleep prob- 
lems account for significant direct (an estimated \$14 billion for insomnia [24]) and indirect costs (sleep-related fatigue alone is estimated to cost businesses $\$ 150$ billion yearly [23]). Poor sleep has an influence on several comorbid conditions including diabetes, obesity and cardiovascular morbidity, significantly affects behavior and has a strong association with psychiatric or psychological conditions. Further, poor sleep can result in impaired cognitive function, decreased quality of life and job performance, and increased risk of road and industrial accidents [23].

Like sleep, mood disorders also have significant financial costs. Annual cost estimates for depression and anxiety disorders are over $\$ 100$ billion [25]. However, the indirect costs and intangible effects of mood-related problems have a much greater societal impact. While negative affect is frequently associated with risk for illness and mortality, positive affect is associated with lower morbidity and improved health outcomes [26], [27]. In this study, we examine the mood and sleep of "healthy" individuals as a part of their everyday life to understand how it affects them.

The connection between mood and social interactions has been made in the past [1], [6] but primarily in individuals with mood and sleep disorders. Also, no connection has been established between sleep and social interactions. Due to the significant financial and societal costs associated with sleep and mood as well as the bidirectional relationship that they share [28], [29], [30], we endeavor to study them together to explore the role that face-to-face interactions might play. The goal of this work is to provide novel insights into these relationships that might provide greater impetus to move from this pilot to more substantial large scale and/or longterm experiments that could ultimately help design useful interventions to improve the everyday living experience. We describe the experiment in the next section, followed by a discussion of the results and the conclusion.

\section{EXPERIMENT}

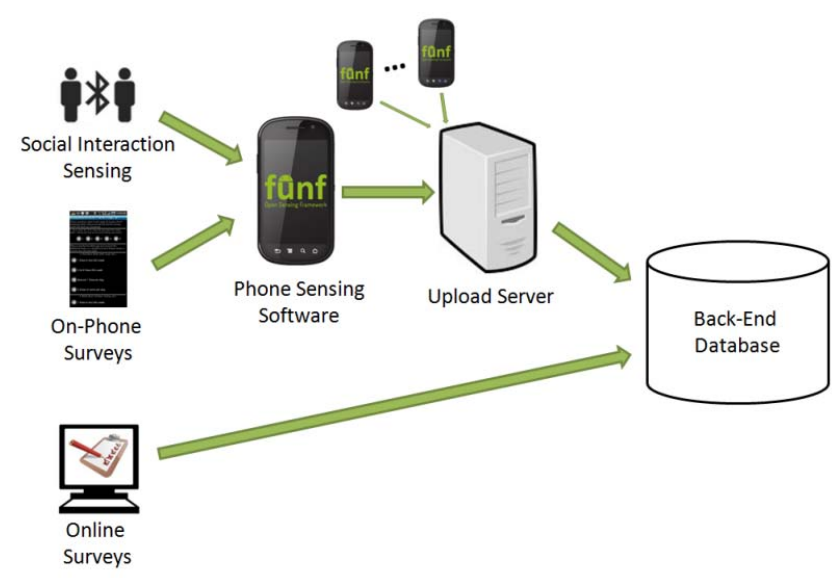

Fig. 1. Overview of the Funf Sensing Platform for Android Smartphones

\section{A. Overview and Participants}

Starting March 2010, we initiated a living laboratory conducted with members of a young-family residential living community adjacent to a major research university in North America, referred to as the Friends and Family Study [31]. All members of the community are couples, and at least one the members is affiliated with the university, usually as a graduate student. The entire community is composed of over 400 residents, approximately half of which have children. The residence has a vibrant community life, with many ties of friendship between its members. The data used for this analysis was collected from 54 participants in the pilot phase of the study, which lasted from March to September 2010. Participants provided informed consent as approved by the Committee on the Use of Humans as Experimental Subjects at our institution.

Study participants were provided Android smartphones with our social and behavioral software sensing platform, called "Funf" 1 that allowed us to track face-to-face interactions through Bluetooth proximity sensing in addition to other behavioral, contextual and communication patterns [31]. The software was responsible for automatically uploading data to the system's backend server whenever Internet access was available. In addition, subjects completed study related surveys at regular intervals. Monthly, daily and weekly surveys include questions about relationships and social activities, personality using standard scales like the Big-Five Inventory [32] and behavioral information such as mood, diet, exercise and sleep. Participants had the option to fill in the surveys either via the phone software, or via a web-based service. Both survey inputs as well as the phone collected data were aggregated in a single back end database for the data relevant to the current analysis.

\section{B. Dataset}

The dataset used in the current analysis is a subset of the Friends and Family dataset which includes a combination of self-reported survey data and automatically captured face-toface interactions. In this work we exclude May and the summer months as many participants were off campus for long periods during these months, and for the current investigation we wanted to evaluate a period where there is the opportunity for dense face-to-face interaction within the study population. Therefore, for the current discussion we analyze the data collected from the 54 healthy subjects over the month of April, 2010. The data included the following signals:

1) Sleep: The sleep information was self-reported by subjects on a daily basis where the options of hours provided included: $<5,5,6,7,8,9,>9$.

2) Mood: Participants were also asked to select their predominant mood for the day from multiple options. The options were ultimately grouped into 'good' mood (Happy or content, Relaxed or peaceful) and 'poor mood' (Stressed or anxious, Angry or frustrated), resulting in a binary variable.

\footnotetext{
${ }^{1}$ http://funf.media.mit.edu/
} 
3) Interactions and Sociability: Face-to-face social interactions are derived from Bluetooth proximity detection data in a manner similar to those in previous reality mining studies [15], [33]. The Funf phone sensing platform, described above, was used to detect Bluetooth devices in the user's proximity. The Bluetooth scan was performed periodically, every five minutes, in order to keep from draining the battery while achieving a high enough resolution for social interaction in a manner. As there are two detections for every five minute period from the two phones in question, each proximity scan is used to represent a 2.5 minute interval as in previous studies [16]. The face-to-face interactions are measured within the study population of 54 participants, all-residing in the same residential complex. While it may become possible in the future to gather a majority of face-to-face interactions with those outside the community as well, it is currently not viable due to the lack of widespread Bluetooth usage necessary to make this possible.

Interaction can be defined as a social encounter between two individuals within this community as detected by Bluetooth proximity scans indicating co-location. Multiple proximity detections on consecutive scans are considered to be part of the same interaction.

Daily sociability can be defined as the total amount of interactions that an individual has had on a particular day (considered to be from 4am on a particular day to 4am on the next to include post-midnight interactions, common in a student community, within the same day). This measure defines how social an individual has been on that day.

This value has been normalized to a value between 0 and 1 , with a value closer to 1 indicating that the sociability on that particular day is close to the highest sociability observed for this individual over the entire period. A value closer to 0 indicates the opposite, the observed daily sociability is close to the lowest observed for that individual. The data was normalized so that high or low sociability is specific to the individual and is not defined by a norm for the community, which could be skewed by a few highly sociable individuals.

Overall sociability can be defined as the total amount of interactions that an individual has had over the one-month period (April 2010). Daily sociability aggregated over time averages out the natural fluctuations of daily sociability to provide a more long-term measure of how social an individual is, during the period under consideration.

These two measures of sociability are defined to bring out the difference between the behavioral effects of an individual's general sociability as opposed to the effects brought out by daily variations. It should be noted that these measures only included face-to-face interactions within this community. Nevertheless, these are assumed to provide a reasonable proxy for sociability as has been the case in previous reality mining studies.

\section{RESULTS}

\section{A. Sleep and Mood}

It is well known that sleep affects mood and vice-versa as discussed earlier. In this work, we explore this bidirectional relationship and find that it holds in this healthy population as well for the one month period. As mood is a binary variable, sleep duration after days in which a participant reported good mood and sleep duration after a participant reported bad mood were separated into two groups and the means of these groups were compared using the non-parametric Wilcoxon Rank Sum test. We find that the means of these two groups are significantly different $(\mathrm{p}=0.0002)$, with the mean dropping from about 7 hours to about 6.4 hours for nights with poor mood. This statistically significant difference suggests that the predominant mood on a particular day might have an effect on that night's sleep.

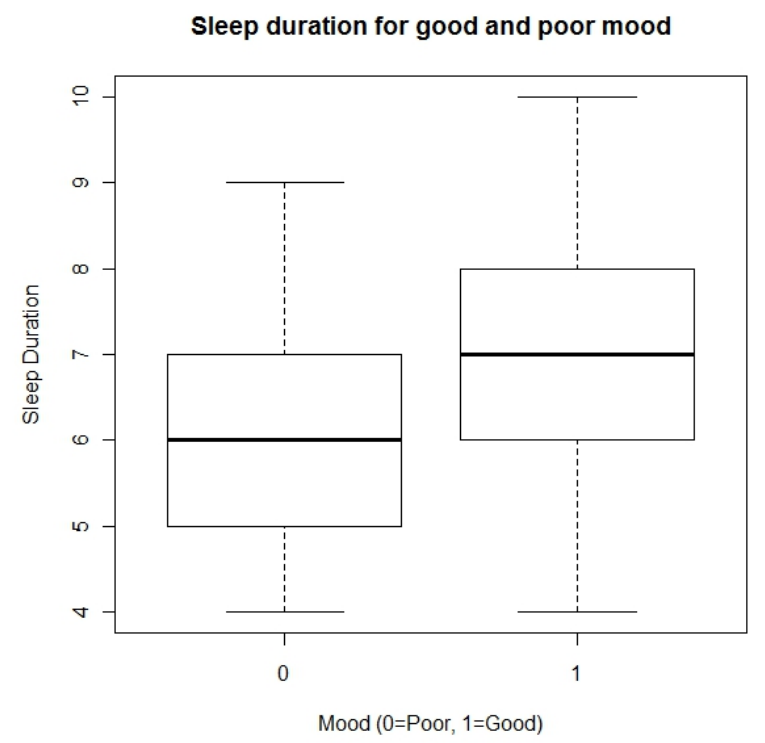

Fig. 2. Sleep duration and mood

As the sleep event occurs after the mood observation in the previous analysis, we are able to study the effect in one direction. Similarly, we test the opposite hypothesis to check whether a night's sleep has an effect on the following day's mood. We find a significant difference $(\mathrm{p}=0.0001)$ between sleep duration to about the same extent, suggesting that this might be true as well. This bidirectional relationship was also detected earlier in this population using a much larger dataset [34]. Figure 2 depicts the difference in sleep duration between days with good mood (6-8 hours in the lower to upper quartiles) and days with poor mood (5-7 hours in the lower to upper quartiles).

\section{B. Mood and Sociability}

Next, we explore the relationship between mood and overall sociability. Using mood information, we separated the groups 
into two: 1) those who exhibited primarily good mood (relaxed, calm, happy, content) i.e., on at least $70 \%$ of the occasions 2) those who exhibited poor mood (stressed, anxious, frustrated, angry) i.e., on at least $30 \%$ of the occasions. The 70-30 breakup is chosen as poor mood is reported much less often than good mood in this community as one might expect. We find that people who fell into the latter group demonstrated significantly lower overall sociability in this community ( $\mathrm{p}=0.02$, Wilcoxon Rank Sum test).

As discussed earlier, it has been noted often that greater social cohesion tends to result in healthier and happier individuals. However, in the past, there was no way to quantify this except through static data collected using surveys, which have several shortcomings [35], particularly in this context. Hence, the result obtained here using a quantified measure of sociability, gathered from interactions throughout the day, lends greater credence to this notion.

In addition to studying the relationship between overall sociability and mood, we also explore the impact of daily sociability on mood. The Wilcoxon Rank Sum test is used again in a manner similar to the analysis for sleep and mood, with sleep duration being replaced by daily sociability. However, no significant difference in daily sociability is observed between days with poor mood and good mood. This may suggest that an individual's changes in sociability may not respond within the same day we are asking about mood. To test for this, we examined the change in daily sociability together with the proportion of days with poor mood, to understand if the variation in sociability is affected by or responds to amount of poor mood. We found no significant correlation and therefore, this result suggests that daily variation in sociability may not be as important as an individual's overall sociability in its effect on mood.

Considering the important role that mood plays in several comorbid conditions, it is important to create interventions that help improve an individual's mood, resulting in positive health effects. If these results were validated through further studies, one group of possible interventions could be targeted towards increasing an individual's overall sociability.

\section{Sleep and Sociability}

Finally, we explore the relationship between sleep and sociability. Lack of sufficient sleep in this community is not surprising. Sources of lack of sleep may include stress of school and children. Approximately 54\% of the couples in this community have children and at least $50 \%$ are students. The overall effect of lack of sufficient sleep in the long-term is evident. However, the effects of short-term lack of sleep are less understood. This is especially true as it relates to things that are affected in our daily lives. One might hypothesize that sociability through its impact on mood might have an important role in affecting sleep or vice-versa.

We explore this hypothesis by first studying the effect of overall sociability on sleep. However, we find no statistically significant association like in the case of mood. On further rumination, this is not an unexpected result, as we would

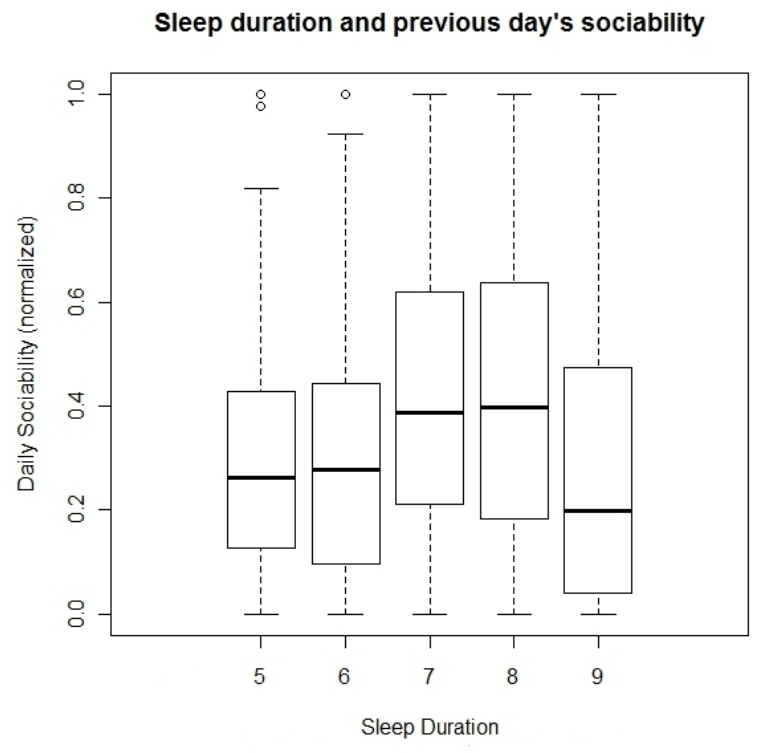

Fig. 3. Sleep duration and previous day's sociability

expect that daily variation in sleep duration is likely to have an association with the variation in daily sociability, if any.

Further analysis reveals that such an association between sleep and daily sociability might indeed exist. Figure 3 depicts the non-linear relationship between daily sociability for a particular day and sleep duration for that night. Several past studies have indicated that a sleep duration of seven to eight hours seems most beneficial while a sleep duration lower than or higher than that is associated with a higher risk of mortality, with the risk increasing as the duration moves further away from the suggested sleep duration [36], [37], [38]. Therefore, it is very interesting to note that daily sociability seems to mirror this trend with highest median sociability on days with a sleep duration of 7 or 8 hours and decreasing on either side with an increase or decrease in sleep duration.

As this relationship is not linear, a Pearson correlation would not be ideal to study this relationship. Hence, we use a Kruskal-Wallis non-parametric analysis of variance to compare daily sociability across the sleep durations ranging from 5 to 9 hours. We find that there is a significant difference in daily sociability for the different sleep durations $(\mathrm{p}<0.001)$.

A visual inspection of figure 3 reveals that the median sociability is much higher for sleep durations 7 and 8 when compared to the other durations. Using Dunn's post hoc test for multiple pairwise comparisons, we find that the difference in daily sociability is significantly different for the following sleep durations: (i) 5 and 7 (ii) 6 and 7 (iii) 6 and 8. This result is intriguing because, to the best of our knowledge, there have been no reports of such an association in the past and such an observation would not have been possible without leveraging the sensing capabilities available today. This fascinating result merits further studies to validate this finding. 
We repeat the analysis to check if the same holds in the other direction. Figure 4 depicts the non-linear relationship sleep duration for a particular night and sleep duration for the following day. While a similar pattern as earlier is observed visually, the Kruskal-Wallis test shows that there is no significant difference across the various sleep durations while comparing the following day's daily sociability. This indicates that the association might hold in one direction only, with daily sociability possibly affecting one's sleep but the opposite not holding true.

\section{Sleep duration and following day's sociability}

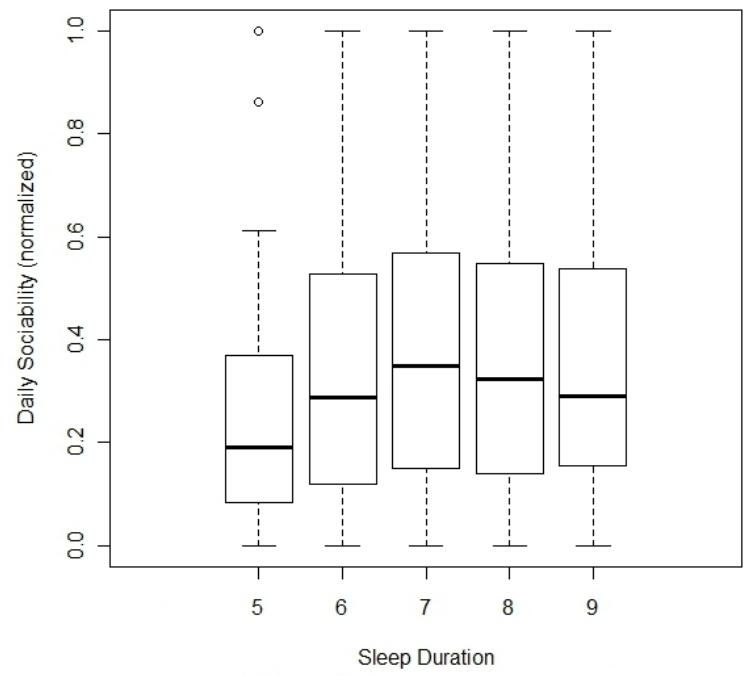

Fig. 4. Sleep duration and following day's sociability

\section{DISCUSSION AND CONCLUSIONS}

In this work, we study several aspects of the relationships between sleep, mood and sociability using data from a population of healthy young couples in a naturalistic setting. At least one spouse in this population is a student and about half the couples have young children. The youngsters hail from a varied backgrounds and ethnicities much like the population of the US. The situations faced by this population in terms of stress and other behavioral factors is not different from other young couples in this age group. These factors make the population of this study more representative of the general populace when compared with previous reality mining studies that included undergraduate students.

In tune with our expectations, some of the characteristics of this data are similar to those observed for the general population. We find that $57.2 \%$ of the reported sleep durations in this data are 7 and 8 hours, with $32.5 \%$ accounting for sleep durations of 6 hours or less and the remaining $10.3 \%$ making up reported sleep durations of 9 hours or more. A histogram of this data is shown in figure 5. This is not very different from sleep durations reported for a cohort of young adults [39]. In addition, we find that the women in this population sleep slightly better than the men, a statistically significant $(p<0.001)$ observation. This is in tune with existing sleep literature [40], which suggests the same, with the difference between the two groups increasing with age. Also, as one might surmise, poor mood is observed less often in general as seen in figure 6 and a smaller number of interactions are more frequently observed as seen in figure 7 .

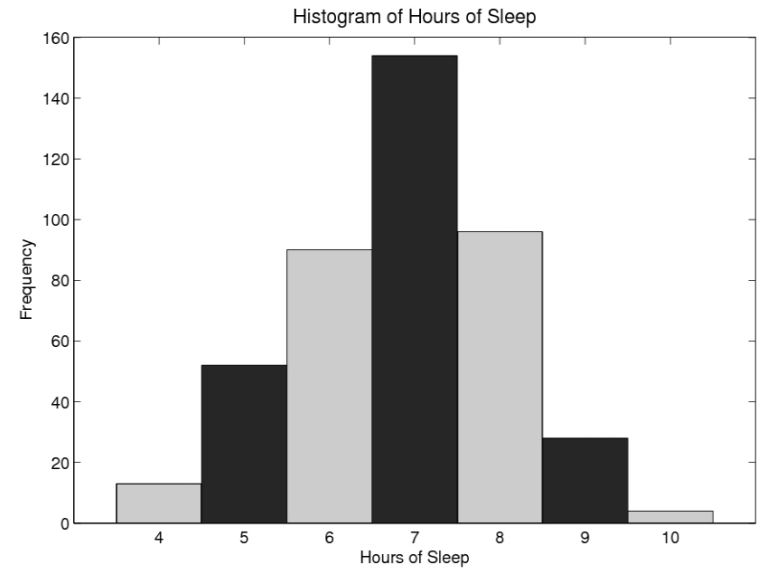

Fig. 5. Sleep in this community

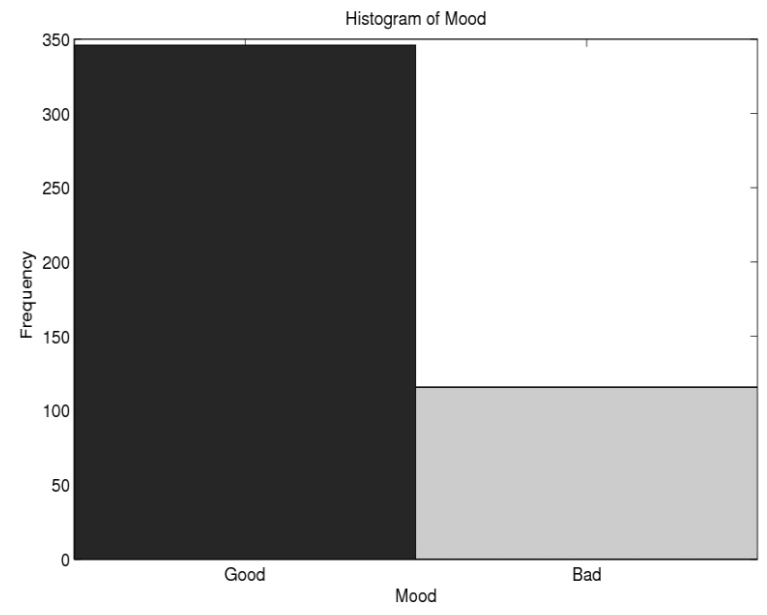

Fig. 6. Mood in this community

Apart from the general characteristics of the data, we were also able to detect a significant bidirectional relationship between sleep and mood, another indicator that the results from such a population might be more generalizable. In particular, this is interesting because this observation has not commonly been made in a naturalistic setting with health young couples.

Next, we detect that individuals with lower overall sociability, a measure of how social an individual was in general, 


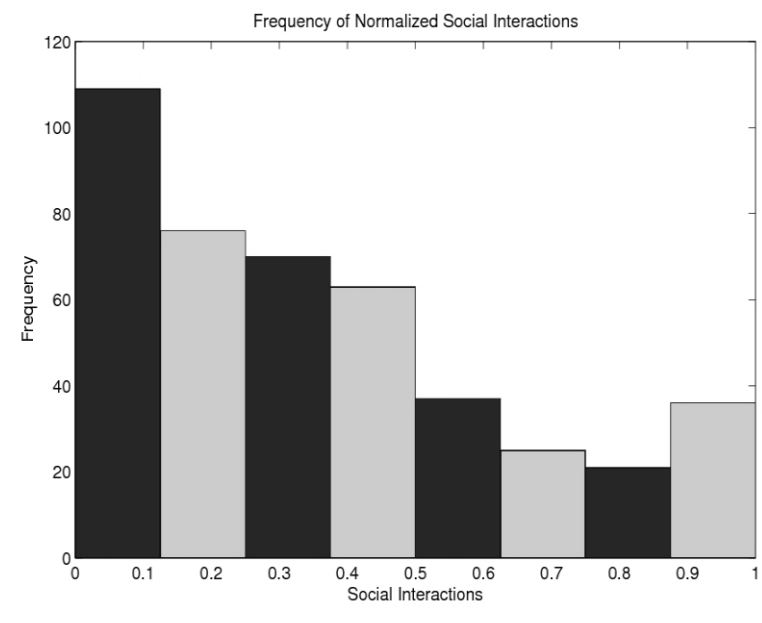

Fig. 7. Sociability in this community

show poor mood more often, a statistically significant result. While such a connection has been made in the past, it has never been observed with quantified social interaction data. This clearly depicts the usefulness of reality mining studies that are able to use phone-based social sensing to gather rich information about dynamic face-to-face interactions.

We also find that an individual's daily sociability, a measure of how social they are on a particular day shows a significant association with that night's sleep duration. Higher median daily sociability is observed during days when the night's sleep duration is in the suggested 7-8 hour range. This is a novel result that has not been reported in the past and would not have been possible without quantifying dynamic social interaction data.

Adding another layer to the notion of social effects, regression analyses from a recent study [34] using data from the same population showed that a subject's sleep and mood might be affected by their spouse's sleep and mood in addition to their own mood and sleep.

These results are important for several reasons. Firstly, reality mining studies have been used to study network phenomenon and behavioral effects in the past. These results indicate their usefulness in understanding phenomenon related to public health, that would not be possible without the use of smartphones as social sensors to quantify the subjects' face-toface interactions. Secondly, the results indicate the importance of social interactions on an individual's daily life and overall well-being, providing further impetus to a burgeoning area of research that has been gaining attention in recent years. Finally, the results while interesting are only early indicators of the potential of such studies. They present a strong reason to move beyond our pilot and pursue larger studies that can validate these notions.

If validated through such larger studies, these results can strongly rejuvenate the case for designing behavioral inter- ventions targeted to improve the quantity and quality of social interactions that an individual has due to their direct and indirect effects of well-being. Much like one needs to watch what they eat and exercise on a regular basis to stay healthy, it might be found that individuals need to watch how and how much they interact in order to stay healthy.

As an attempt to move in this direction, we are currently running an experiment gathering rich sleep data (quantifying sleep quality in addition to quantity) using a wireless sensing headband (a commercial product manufactured by Zeo Inc.) to leverage the opportunity of studying sleep, mood and sociability in this naturalistic setting with far richer data for sleep and mood as well. In conclusion, we hope that this work bring greater attention to the potential of reality mining and the novel applications of wireless sensing for directions such as public health research.

\section{ACKNOWLEDGMENT}

We would like to thank Dr. Stuart Quan from the Division of Sleep Medicine at Harvard Medical School for guiding us on aspects related to Sleep. We would like to thank Cory Ip for significant contributions towards experimental deployment and management. We would also like to thank several undergraduate contributors for their help with the development of the sensing platform. This work was partially sponsored by the Army Research Laboratory under Cooperative Agreement Number W911NF-09-2-0053, and by AFOSR under Award Number FA9550-10-1-0122. This work was also partially sponsored by the MIT/Masdar Collaborative Research Grant. Views and conclusions in this document are those of the authors and should not be interpreted as representing the official policies, either expressed or implied, of the ARL, AFOSR, or the U.S. Government.

\section{REFERENCES}

[1] L. Berkman, "Assessing the physical health effects of social networks and social support," Annual Review of Public Health, vol. 5, no. 1, pp. 413-432, 1984.

[2] — "The role of social relations in health promotion," Psychosomatic Medicine, vol. 57, no. 3, p. 245, 1995.

[3] L. Berkman, T. Glass, I. Brissette, and T. Seeman, "From social integration to health: Durkheim in the new millennium," Social Science \& Medicine, vol. 51, no. 6, pp. 843-857, 2000.

[4] M. Marmot and R. Wilkinson, Social determinants of health. Oxford University Press, 2005.

[5] S. Cohen, B. H. Gottlieb, and L. G. Underwood, "Social relationships and health," American Psychologist, vol. 59, pp. 676-684, 2004.

[6] K. Rook, "The negative side of social interaction: Impact on psychological well-being." Journal of Personality and Social psychology, vol. 46, no. 5, p. 1097, 1984.

[7] P. Bellair, "Social interaction and community crime: Examining the importance of neighbor networks," Criminology, vol. 35, p. 677, 1997.

[8] E. Glaeser, B. Sacerdote, and J. Scheinkman, "Crime and social interactions," 1995.

[9] H. Hong, J. Kubik, and J. Stein, "Social interaction and stock-market participation," The Journal of Finance, vol. 59, no. 1, pp. 137-163, 2004.

[10] E. Beiseitov, J. Kubik, and J. Moran, "Social interactions and the health insurance choices of the elderly: Evidence from the health and retirement study," Syracuse University, Maxwell School for Citizenship and Public Affairs, Center for Policy Research, Working Paper, 2004.

[11] N. Christakis and J. Fowler, "The spread of obesity in a large social network over 32 years," New England Journal of Medicine, vol. 357, no. 4 , p. $370,2007$. 
[12] S. Mednick, N. Christakis, and J. Fowler, "The spread of sleep loss influences drug use in adolescent social networks," PLoS One, vol. 5, no. 3, p. e9775, 2010.

[13] D. Roy, R. Patel, P. DeCamp, R. Kubat, M. Fleischman, B. Roy, N. Mavridis, S. Tellex, A. Salata, J. Guinness, M. Levit, and P. Gorniak, "The human speechome project," Lecture Notes in Computer Science, vol. 4211, p. 192, 2006.

[14] N. Eagle and A. Pentland, "Social network computing," Lecture notes in computer science, pp. 289-296, 2003.

[15] —_ "Reality mining: sensing complex social systems," Personal and Ubiquitous Computing, vol. 10, no. 4, pp. 255-268, 2006.

[16] N. Eagle, A. Pentland, and D. Lazer, "Inferring friendship network structure by using mobile phone data," Proceedings of the National Academy of Sciences, vol. 106, no. 36, p. 15274, 2009.

[17] T. Choudhury, M. Philipose, D. Wyatt, and J. Lester, "Towards activity databases: Using sensors and statistical models to summarize peoples lives," IEEE Data Eng. Bull, vol. 29, no. 1, pp. 49-58, 2006.

[18] D. Olguin Olguin, P. Gloor, and A. Pentland, "Wearable Sensors for Pervasive Healthcare Management," 2009.

[19] D. Olguin Olguin, B. Waber, T. Kim, A. Mohan, K. Ara, and A. Pentland, "Sensible organizations: Technology and methodology for automatically measuring organizational behavior," IEEE Transactions on Systems, Man, and Cybernetics-B, 2009.

[20] A. Madan, S. Moturu, D. Lazer, and A. Pentland, "Social sensing: obesity, unhealthy eating and exercise in face-to-face networks," in Wireless Health 2010. ACM, 2010, pp. 104-110.

[21] A. Madan, M. Cebrian, S. Moturu, K. Farrahi, and A. Pentland, "Sensing the health state of a community," IEEE Pervasive Computing, forthcoming.

[22] A. Madan, M. Cebrian, D. Lazer, and A. Pentland, "Social sensing for epidemiological behavior change," in Proceedings of the 12th ACM international conference on Ubiquitous computing. ACM, 2010, pp. 291-300.

[23] H. Colten and B. Altevogt, Sleep disorders and sleep deprivation: an unmet public health problem. National Academies Press, 2006.

[24] J. Walsh, "Clinical and socioeconomic correlates of insomnia." Journal of Clinical Psychiatry, vol. 65, no. 8, pp. 13-19, 2004.

[25] R. Kessler, P. Greenberg et al., "The economic burden of anxiety and stress disorders," Neuropsychopharmacology: The Fifth Generation of Progress. Baltimore, MD: Lippincott Williams and Wilkins, pp. 981992, 2002.

[26] S. Cohen and S. Pressman, "Positive affect and health," Current Directions in Psychological Science, vol. 15, no. 3, p. 122, 2006.

[27] A. Steptoe, K. O'Donnell, M. Marmot, and J. Wardle, "Positive affect, psychological well-being, and good sleep," Journal of psychosomatic research, vol. 64, no. 4, pp. 409-415, 2008.
[28] R. Benca, M. Okawa, M. Uchiyama, S. Ozaki, T. Nakajima, K. Shibui, and W. Obermeyer, "Sleep and mood disorders," Sleep Medicine Reviews, vol. 1, no. 1, pp. 45-56, 1997.

[29] M. Peterson and R. Benca, "Sleep in mood disorders." Psychiatric Clinics of North America, 2006.

[30] D. Ford and L. Cooper-Patrick, "Sleep disturbances and mood disorders: an epidemiologic perspective," Depression and Anxiety, vol. 14, no. 1, pp. 3-6, 2001

[31] N. Aharony, W. Pan, C. Ip, I. Khayal, and A. Pentland, "The social fmri: Measuring, understanding and designing social mechanisms in the real world," in Proceedings of the 13th ACM International Conference on Ubiquitous Computing, 2011.

[32] O. John and S. Srivastava, "The big five trait taxonomy: History, measurement, and theoretical perspectives," Published as, 1999.

[33] A. Madan and A. Pentland, "Modeling social diffusion phenomena using reality mining," in Proceedings of AAAI Spring Symposium on Human Behavior Modeling 2009, 2009.

[34] S. Moturu, I. Khayal, N. Aharony, and A. Pan, W. Pentland, "Sleep, mood and sociability in a healthy population," in Proceedings of the 33rd Annual International Conference of the IEEE Engineering in Medicine and Biology Society, 2011.

[35] S. Sudman and N. Bradburn, "Effects of time and memory factors on response in surveys," Journal of the American Statistical Association, vol. 68 , no. 344 , pp. $805-815,1973$.

[36] P. Heslop, G. Smith, C. Metcalfe, J. Macleod, and C. Hart, "Sleep duration and mortality: the effect of short or long sleep duration on cardiovascular and all-cause mortality in working men and women," Sleep medicine, vol. 3, no. 4, pp. 305-314, 2002.

[37] D. Kripke, L. Garfinkel, D. Wingard, M. Klauber, and M. Marler, "Mortality associated with sleep duration and insomnia," Archives of general psychiatry, vol. 59, no. 2, pp. 131-136, 2002.

[38] S. Patel, N. Ayas, M. Malhotra, D. White, E. Schernhammer, F. Speizer, M. Stampfer, and F. Hu, "A prospective study of sleep duration and mortality risk in women," Sleep, vol. 27, no. 3, pp. 440-444, 2004.

[39] A. Steptoe, V. Peacey, and J. Wardle, "Sleep duration and health in young adults," Archives of internal medicine, vol. 166, no. 16, p. 1689, 2006.

[40] E. Bixler, M. Papaliaga, A. Vgontzas, H. Lin, S. Pejovic, M. Karataraki, A. Vela-Bueno, and G. Chrousos, "Women sleep objectively better than men and the sleep of young women is more resilient to external stressors: effects of age and menopause," Journal of Sleep Research, vol. 18, no. 2, pp. 221-228, 2009. 